\title{
Human colonic in vitro fermentation of water-soluble arabinoxylans from hard and soft wheat alters Bifidobacterium abundance and short-chain fatty acids concentration
}

\author{
Candela Paesani $^{\text {a }}$, Lorena S. Sciarini ${ }^{\text {a }}$, Malena Moiraghi ${ }^{\mathrm{a}, \mathrm{b}}$, Emiliano Salvucci ${ }^{\mathrm{a}}$, Samira B. \\ R. Prado ${ }^{c}$, Gabriela Teresa Pérez ${ }^{\mathrm{a}, \mathrm{b}}$, João Paulo Fabi ${ }^{\mathrm{c}, \mathrm{d}, \mathrm{e}, *}$ \\ ${ }^{a}$ ICYTAC (Instituto de Ciencia y Tecnología Córdoba), CONICET-UNC, Córdoba, Argentina \\ ${ }^{\mathrm{b}}$ Cátedra de Química Biológica, Facultad de Ciencias Agropecuarias, UNC, Córdoba, Argentina \\ ${ }^{\mathrm{c}}$ Department of Food Science and Experimental Nutrition, School of Pharmaceutical Sciences, University of São Paulo, São Paulo, SP, Brazil \\ ${ }^{\mathrm{d}}$ Food and Nutrition Research Center (NAPAN), University of São Paulo, São Paulo, SP, Brazil \\ ${ }^{\mathrm{e}}$ Food Research Center (FoRC), CEPID-FAPESP (Research, Innovation and Dissemination Centers, São Paulo Research Foundation), São Paulo, SP, Brazil
}

\section{A R T I C L E I N F O}

\section{Keywords:}

Arabinoxylans

Human gut microbiota

Bifidobacterium

Short-chain fatty acid

Wheat

\begin{abstract}
A B S T R A C T
The human intestinal microbiome plays an important role in health due to the large number of beneficial effects related to the bacterial profile and the metabolites generated in the intestine. Arabinoxylans are compounds present in different cereals such as wheat and they can modulate the profile and functioning of some beneficial bacteria from human intestinal microbiota. In the present work, a colonic in vitro fermentation with human faecal inoculum was done using arabinoxylans extracted from Argentinian hard and soft wheat as substrates. Molecular size alteration of arabinoxylans were studied during fermentation and Lactobacillus and Bifidobacterium abundance as well as short chain fatty acids concentrations were determined. The arabinoxylans fermentation was proved to induce the growth of Bifidobacterium and the release of short-chain fatty acids. The speed and efficiency of fermentation were different for each of the arabinoxylan extracted from both wheat genotypes, perhaps because of differences in their chemical and physical structures. The consumption of water-extractable arabinoxylans (WE-AX) (either supplemented or enriched) to maintain the balance or modulate in a favorable way the profile of Bifidobacterium can be an important contribution to the human health.
\end{abstract}

\section{Introduction}

The human microbiome is the total population of microorganisms and metabolites which colonise the whole human body (Petrosino, Highlander, Luna, \& Gibbs, 2009). The gut microbiota is one of the most densely populated communities (Ruiz Álvarez, Puig Peña, \& Rodríguez Acosta, 2010). In the past several years, more attention has been given to gut microbiota, and several studies have shown modulating functions on different aspects of the digestive function (Salvucci, 2014). In more recent years, the intestinal microbiome has been considered a symbiotic partner for health maintenance (Qin et al., 2010). The intestinal microbiome defines and modulates host homeostasis by determining nutritional, immunological, and neuroendocrine balance (Bakhtiar et al., 2013; Salvucci, 2014). The nutrients which are not digested by human enzymes, such as nondigestible carbohydrates, reach the intestine and can be fermented by the gut microbiota, obtaining final products such as short-chain fatty acids (SCFA) (Karppinen, Liukkonen, Aura, Forssell, \& Poutanen, 2000; Kasubuchi, Hasegawa, Hiramatsu, Ichimura, \& Kimura, 2015).

Prebiotics are compounds which improve human health and reduce risk of diseases mediated by microbiota alterations (Gibson et al., 2017). Those compounds which are selectively fermented by gut bacteria induce specific changes in the composition and/or activity of the gastrointestinal microbiome, which confers benefits to the welfare of the host and health in general (Al-sheraji, Ismail, Yazid, \& Mustafa, 2013; Gibson et al., 2017; Gibson \& Fuller, 2000). The prebiotic effect in the intestine can be assessed by the enhanced proliferation of Bifidobacterium and Lactobacillus (Gibson, 1999), the decreased amount of intestinal pathogens, the increased production of metabolites related to the activity of beneficial bacteria such as SCFA, and the decrease in the

\footnotetext{
* Corresponding author. ICYTAC (Instituto de Ciencia y Tecnología Córdoba), CONICET-UNC, Córdoba, Argentina.

E-mail address: jpfabi@usp.br (J.P. Fabi).
} 
Table 1

pH and pressure values during fermentation. ${ }^{\mathrm{a}}$

\begin{tabular}{llll}
\hline$<!-C o l$ Count:4- $>$ Time (h) & Samples & $\mathrm{pH}^{\mathrm{b}}$ & Pressure (psi) \\
\hline 0 & Control & $9.0 \pm 0.5 \mathrm{a}$ & $0.0 \pm 0.1 \mathrm{a}$ \\
& Inulin & $9.0 \pm 0.5 \mathrm{a}$ & $0.0 \pm 0.1 \mathrm{a}$ \\
& AXs & $9.0 \pm 0.5 \mathrm{a}$ & $0.0 \pm 0.1 \mathrm{a}$ \\
& AXh & $9.0 \pm 0.5 \mathrm{a}$ & $0.0 \pm 0.1 \mathrm{a}$ \\
6 & Control & $8.2 \pm 0.3 \mathrm{a}$ & $1.0 \pm 0.1 \mathrm{a}$ \\
& Inulin & $6.2 \pm 0.2 \mathrm{~b}$ & $1.0 \pm 0.3 \mathrm{a}$ \\
& AXs & $7.5 \pm 0.2 \mathrm{a}$ & $1.0 \pm 0.4 \mathrm{a}$ \\
12 & AXh & $7.5 \pm 0.1 \mathrm{a}$ & $1.2 \pm 0.3 \mathrm{a}$ \\
& Control & $7.1 \pm 0.1 \mathrm{a}$ & $1.3 \pm 0.2 \mathrm{a}$ \\
& Inulin & $5.8 \pm 0.2 \mathrm{~b}$ & $2.5 \pm 0.3 \mathrm{~b}$ \\
24 & AXs & $6.2 \pm 0.1 \mathrm{~b}$ & $3.5 \pm 0.3 \mathrm{c}$ \\
& AXh & $5.8 \pm 0.3 \mathrm{~b}$ & $4.2 \pm 0.3 \mathrm{~d}$ \\
& Control & $6.7 \pm 0.2 \mathrm{c}$ & $2.1 \pm 0.2 \mathrm{a}$ \\
& Inulin & $5.3 \pm 0.4 \mathrm{a}$ & $4.0 \pm 0.3 \mathrm{~b}$ \\
& AXs & $6.0 \pm 0.4 \mathrm{~b}$ & $5.3 \pm 0.1 \mathrm{c}$ \\
& AXh & $5.7 \pm 0.3 \mathrm{ab}$ & $7.2 \pm 0.4 \mathrm{~d}$ \\
& Control & $6.2 \pm 0.5 \mathrm{~b}$ & $2.0 \pm 0.3 \mathrm{a}$ \\
& Inulin & $5.2 \pm 0.2 \mathrm{a}$ & $5.0 \pm 0.1 \mathrm{~b}$ \\
& AXs & $5.1 \pm 0.1 \mathrm{a}$ & $5.0 \pm 0.1 \mathrm{~b}$ \\
& AXh & $5.3 \pm 0.3 \mathrm{a}$ & $7.0 \pm 0.1 \mathrm{c}$ \\
\hline
\end{tabular}

$\mathrm{b}$ The results are expressed as mean average and standard error of a triplicate. Different letters in the columns mean significant differences $(p<0.05)$.

${ }^{\mathrm{a}}$ Variation of pressure and $\mathrm{pH}$ during the $28 \mathrm{~h}$ of the fermentation test.

production of toxic metabolites (Al-sheraji et al., 2013).

Arabinoxylans (AX) are nondigestible carbohydrates considered as the main non-cellulosic polysaccharides in the cell walls of cereals such as wheat (Paesani, Salvucci, Moiraghi, Fernandez Canigia, \& Pérez, 2019). The AX present in wheat are classified according to their solubility in water: soluble (water-extractable AX - WE-AX) or insoluble; between 25 and $33 \mathrm{~g} / 100 \mathrm{~g}$ of wheat AX are WE-AX (Saulnier, Sado, Branlard, Charmet, \& Guillon, 2007). AX are formed by xylose chains linked by a $\beta 1-4$ bond, substituted by arabinose units linked by $1 \alpha-2$ and $1 \alpha-3$ bonds along the xylose chain (Grootaert et al., 2009; Mendis \& Simsek, 2015). Ferulic acid can be attached at the C(O)-5 position of the arabinose. It is still not clear how the proportions of the number and type of substitution influence the heterogeneous nature of the AX (Saulnier et al., 2007).

The structural properties of AX determine their biological effects, fermentation processes, and modulation of gut microbiota. Some studies have reported the effect of molecular structure on the functionality of $\mathrm{WE}-\mathrm{AX}$, such as the lower the molecular weight of WE-AX is, the higher the prebiotic effect is (Van Craeyveld et al., 2008). The distinct fermentation effects of WE-AX could be due to AX structure variation depending on the plant tissue components and wheat variety (Sun, Sun, \& Wang, 2017). For instance, hard and soft wheat produces flours with different particle sizes when milled, which can result in different WE-AX structures. However, there are limited reports regarding colonic fermentation of hard and soft WE-AX and their possible prebiotic effects. Thus, the aim of this work is to investigate the effect of WE-AX extract from hard and soft wheat on modulation of human gut microbiota in a colonic in vitro fermentation by analysing Lactobacillus and Bifidobacterium populations, SCFA production, and WE-AX molecular size changes during the fermentation, using inulin as a positive prebiotic control.

\section{Material and methods}

\subsection{Extraction of WE-AX from hard and soft wheat}

Two pools of water-extractable AX (WE-AX) of wheat were obtained: one pool of soft Argentinian wheat (AXs) (experimental lines identified by the acronym \#PM carrying the alleles Pina-D1 a/Pinb-D1 a; triplicate), and another of hard wheat (AXh) (commercial Argentinian cultivars Klein Yarara, ACA 315, and Guerrero; triplicate). The WE-AX extraction was done according to Buksa et al. (2010), with modification (Paesani et al., 2020). The AX samples were extracted with water $\left(12 \mathrm{~h}\right.$ at $\left.50{ }^{\circ} \mathrm{C}\right)$ and then centrifuged $(1250 \times g, 10 \mathrm{~min})$. The supernatant was treated with thermo-resistant $\alpha$-amilase (Sigma, $350 \mathrm{kU}$ ) and pronase E (from Streptomyces griseus, Sigma Type XIV $3.5 \mathrm{U} / \mathrm{mg}$ ), the solution was filtered and the WE-AX precipitated with acetone:alcohol (1:1). Finally, the extract was dried in an oven for $2 \mathrm{~h}$ at $50{ }^{\circ} \mathrm{C}$. The characterization of the samples is depicted in Table S1 of the supplementary material and was discussed in detail by Paesani et al. (2020).

\subsection{Colonic batch in vitro fermentation}

The in vitro fermentation of the WE-AX samples was evaluated using the faecal content of three volunteers older than 18 years with no history of gastrointestinal diseases and who did not use antibiotics in the three months prior to the sample collection. This stage was undertaken with the approval of the Research Ethics Committee of the Faculty of Pharmaceutical Sciences of the University of São Paulo (Ethics Committee approval CAAE \#43129115.7.0000.0067, approval \#1.089.446). The faeces were collected in sterile containers and placed on the ice until the sample was delivered. After the reception, they were conditioned in $\mathrm{CO}_{2}$ for maintenance of anaerobiosis. Samples from the three individuals were mixed and reconditioned in $\mathrm{CO}_{2}$. Faecal fermentation was performed as described by (Jonathan, Wiechen, Souza, Schols, \& Gruppen, 2012; M. T.; Williams \& Hord, 2005). The fermentation medium was composed of basal solution $(76 \mathrm{~mL} / 100 \mathrm{~mL})$, vitamin/phosphate solution $(1 \mathrm{~mL} / 100 \mathrm{~mL})$, reducing solution $(1 \mathrm{~mL} / 100 \mathrm{~mL})$, and bicarbonate solution $(4 \mathrm{~mL} / 100 \mathrm{~mL})$ (Williams, Bosch, Boer, Verstegen, \& Tamminga, 2005). Immediately after making the pool, the faeces were diluted six times (w/v) in sterile $\mathrm{NaCl} 0.9 \mathrm{~g} / 100 \mathrm{~mL}$ and were homogenised and filtered in Miracloth (EMD Millipore). One hundred mg of WE-AX were used for the treatment, and inulin was used as a positive control in the same amount. Each weight was corrected for the purity of each extract (Table S1). A control experiment was done using the medium and the inoculum without added fibre and a negative control was done using only medium to guarantee aseptic handling. The bottles with $0.750 \mathrm{~mL}$ of the inoculum, in triplicate, were placed in a $37^{\circ} \mathrm{C}$ bath with shaking, and $50 \mu \mathrm{L}$ samples were taken at $0,6,12,24$, and $28 \mathrm{~h}$. Kinetics of fermentation were assessed by measuring the medium $\mathrm{pH}$ variation and the pressure of the gas production over time point. The pressure of gas production was measured by the apparatus of Theodorou, Williams, Dhanoa, Mcallan, and France (1994) in each fermentation time.

\subsection{Quantification of short-chain fatty acids}

The collected aliquots from each fermentation vessels were centrifuged, and filtered in a $0.22 \mu \mathrm{m}$ filter pore for the determination of SCFA at the times $0,6,12,24$, and $28 \mathrm{~h}$. The quantification was done by gas chromatography (CG Agilent Technologies 7890 B GC System, USA) with flame ionizer detector (FID) using a fused silica column CP 7747 (WCTO, Varian, Palo Alto, CA, USA), under the same protocol as Paesani et al. (2020). The injection was carried out at $250^{\circ} \mathrm{C}$ and $2,8 \mathrm{KPa}$. The initial temperature was $110^{\circ} \mathrm{C}$ followed by an increase of $2{ }^{\circ} \mathrm{C} / \mathrm{min}$ up to $140{ }^{\circ} \mathrm{C}$ and then $40{ }^{\circ} \mathrm{C} / \mathrm{min}$ up to the final temperature of $200{ }^{\circ} \mathrm{C}$. The total SCFA was calculated by adding the total of the acids measured.

\subsection{Molecular size distribution}

Aliquots of the fermentation were collected for the analysis of the molecular size distribution of the polysaccharides at each time point. High pressure molecular size exclusion chromatography coupled with a refractive index detector (HPSEC-RID) was performed using the Infinity 1250 System (Agilent, Santa Clara, CA, USA). The determinations were made as specified in Paesani et al. (2020). 

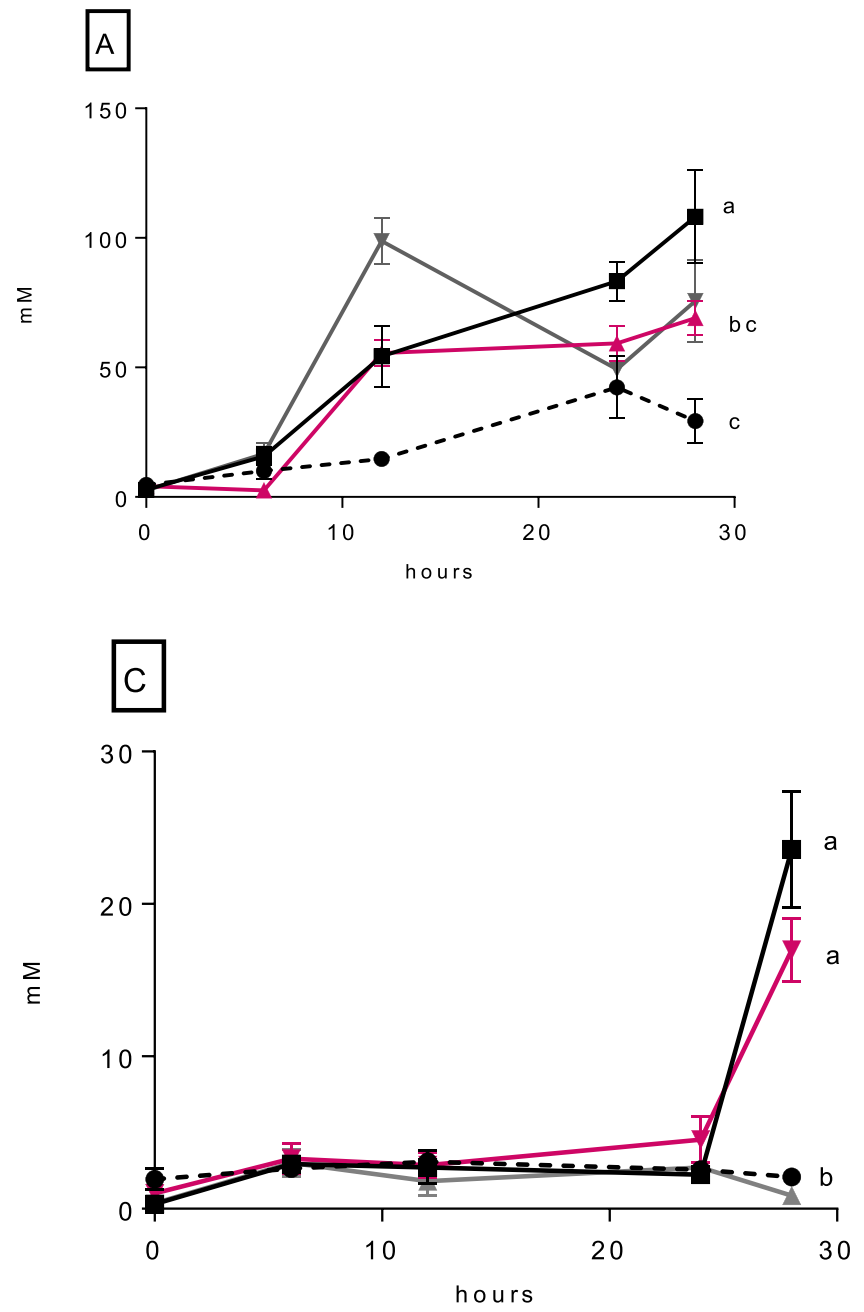

B
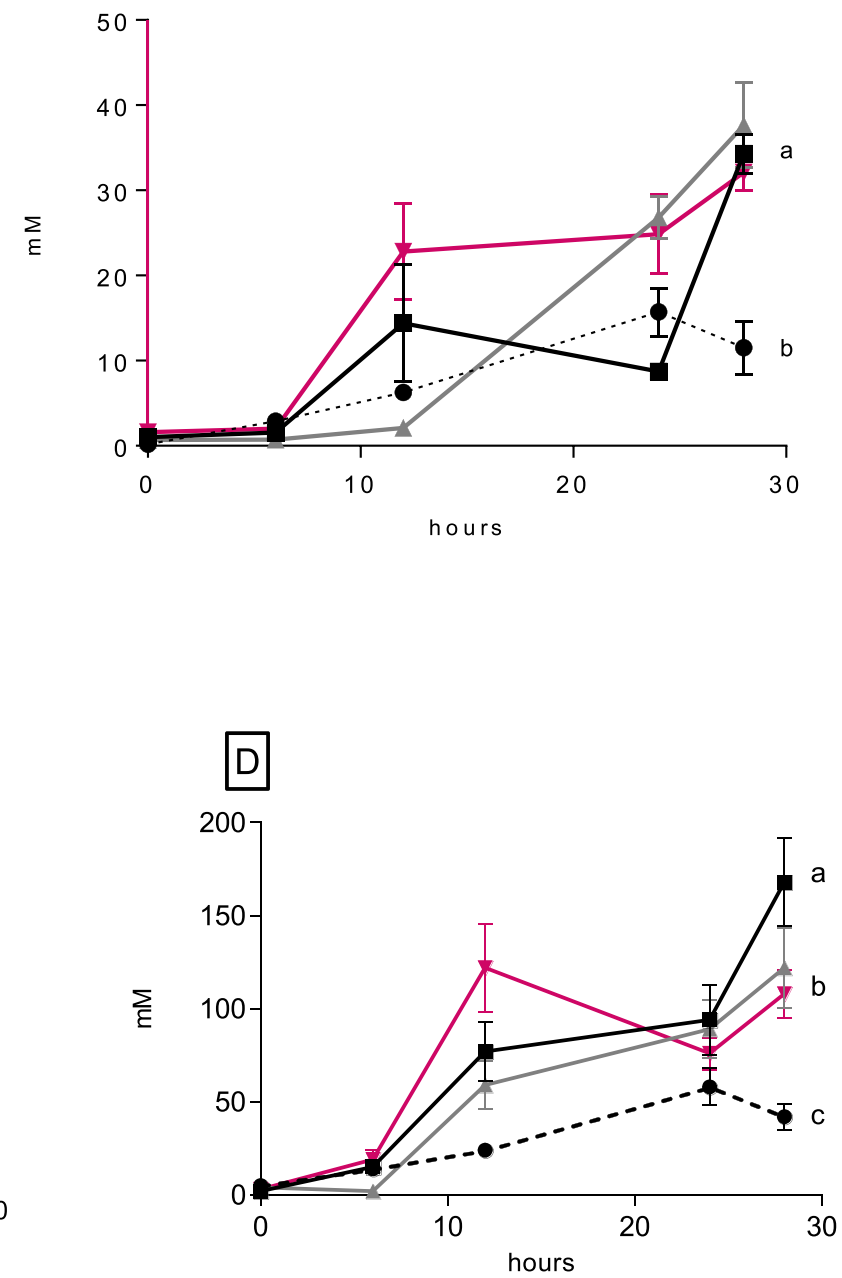

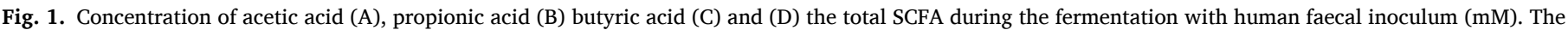

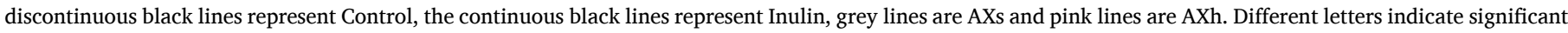

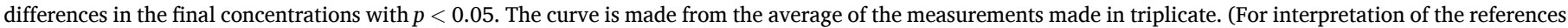
to colour in this figure legend, the reader is referred to the Web version of this article.)

\subsection{Bifidobacterium and Lactobacillus analysis}

After $28 \mathrm{~h}$ of fermentation (inefficacy of fermentation medium), the samples were centrifuged, and DNA was extracted from the precipitate for the quantification of Bifidobacterium and Lactobacillus by qPCR. The whole process was carried out following exactly the protocol detailed by Paesani et al. (2020). Bacterial groups were expressed as a number of copies for $\mu \mathrm{L}$ of the cDNA.

\subsection{Statistical analysis}

The data collected from biological and technical triplicates in experiments were treated statistically by analysis of variance (ANOVA), and the results were compared by the Fisher Method at a significance level of 0.05 . The values were reported as the arithmetic mean, and each letter indicates significant differences. These analyses were performed using the INFOSTAT software (School of Agricultural Sciences, UNC, Córdoba, Argentina).

\section{Results and discussion}

\subsection{AX colonic in vitro fermentation}

We obtained the WE-AX from hard and soft Argentinian wheat, evaluated its composition, and performed colonic in vitro fermentation to evaluate the WE-AX prebiotic potential. The characterisation of WEAX extracts was analysed in detail by Paesani et al. (2020) and is shown in Table 1 of the supplementary material. The pressure increased significantly during the fermentation for both AX extracts and showed greater values when compared to control sample $(2.0 \mathrm{psi})$ at $28 \mathrm{~h}$ (Table 1). Since the human inoculum might have unfermented saccharides, it was expected a subtle increase in pressure and decreased $\mathrm{pH}$ during fermentation in control group. The negative control experiment did not change any parameter.

AXh showed the higher increase in pressure (7.0 psi) even when compared with the prebiotic control inulin (5 psi). The $\mathrm{pH}$ decreased in all samples, reaching the lowest values at $28 \mathrm{~h}$, when fermented medium was inefficient to maintain fermentation (Table 1). There was no significant difference amongst AXh, AXs, and inulin. The control had a final $\mathrm{pH}$ value of 6.2 , possible due to fermentation of reminiscent 
A

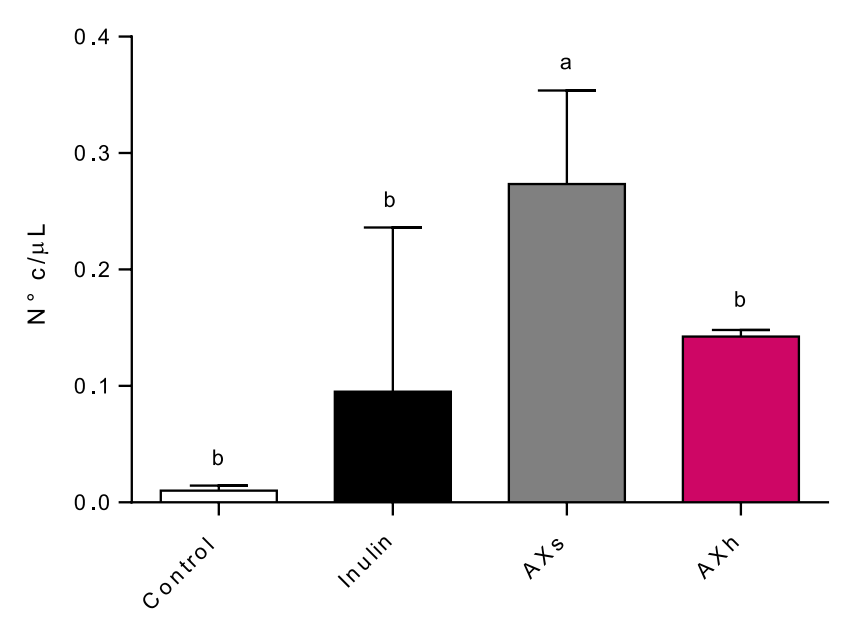

B

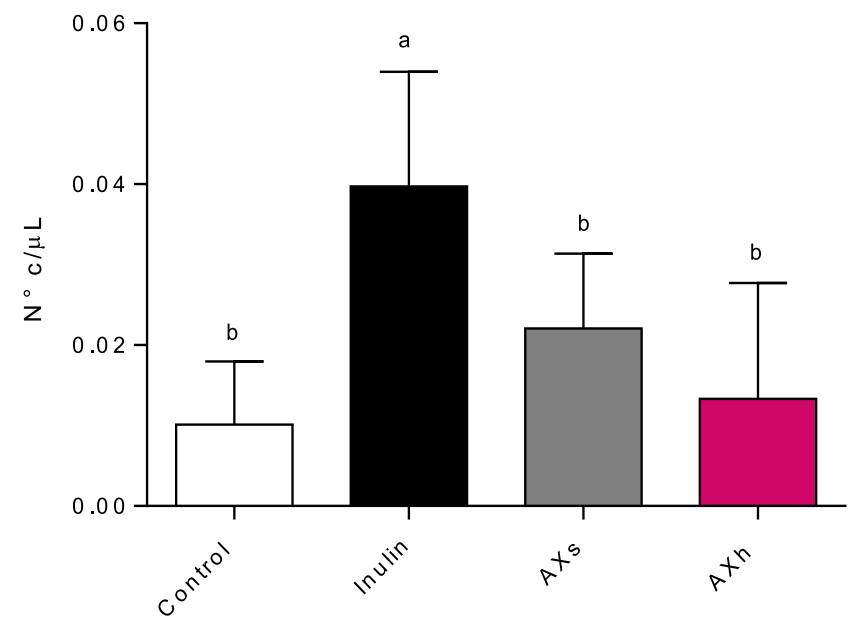

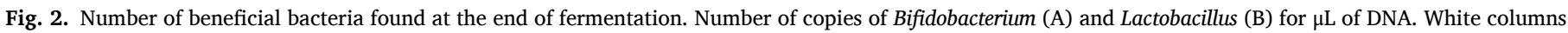

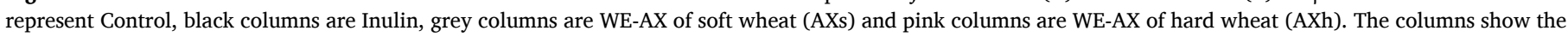

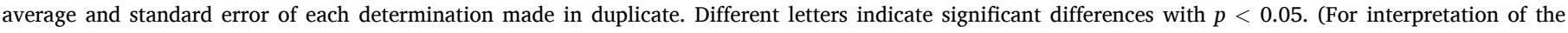
references to colour in this figure legend, the reader is referred to the Web version of this article.)

carbohydrates in human faeces, whilst the WE-AX samples and inulin reached values between 5.1 and 5.3. The colonic $\mathrm{pH}$ decrease is caused by the accumulation of SCFA, a biological health consequence which could decrease the solubility of free bile acids, for example (Grubben et al., 2001). The decrease in $\mathrm{pH}$, therefore, would not only demonstrate the ability of the human inoculum, partially represented by the gut microbiota, to metabolise the WE-AX, thus generating SCFA, but it would also exert a protective effect in the intestine since the acidification inhibits most opportunistic pathogens (Roberfroid, Loo, \& Gibson, 1998, pp. 11-19).

\subsection{Short-chain fatty acids and gas produced during fermentation}

The consequence of polysaccharides fermentation is the production of some types of gasses and SCFA (Topping \& Clifton, 2001). The variation in SCFA concentrations was evaluated at each in vitro fermentation time point. The SCFA are a source of energy for the intestinal epithelial cells, and they could also modulate cells growth. The SCFA also modulate enzymatic activity and transcription factors (Corrêa-Oliveira, Fachi, Vieira, Sato, \& Vinolo, 2016; Kasubuchi et al., 2015). The production of these metabolites is determined by numerous factors, including the amount and type of bacteria present in the colon and the substrate source (Roberfroid, 2007). Thus, the decreased values of the medium $\mathrm{pH}$ are a direct consequence of SCFA formation (Sun et al., 2017). The total production of SCFA was significantly higher for both WE-AX extracts and inulin than control (Fig. 1D).

Acetic acid concentration increased throughout the fermentation for all samples, except for the AXs which showed the highest concentration at $12 \mathrm{~h}$ and then decreased until the end of the fermentation (Fig. 1A). This could be due to a faster fermentation of AXs than AXh. Nevertheless, both WE-AX showed significantly higher values of C:2 than the control. Acetic acid usually has the faster and higher production (Rumpagaporn et al., 2015) and it has been associated with changes in the expression profile of regulatory neuropeptides, favouring appetite suppression and activation of acetyl-CoA carboxylase (Sun et al., 2017).

The fermentation of AX also produced high amounts of propionic acid compared to other studies (Van Laar, Tamminga, Williams, \& Verstegen, 2002; Rose, Inglett, \& Liu, 2010). It increased during the experiment with higher concentrations observed for the WE-AX and inulin. At $12 \mathrm{~h}$ fermentation, the AXs had a lower concentration than control with a sharp increase after. At $12 \mathrm{~h}$, propionic acid in AXh was the highest and then increased progressively (Fig. 1B). Rumpagaporn et al. (2015) have also observed that the propionic acid increased during the fermentation of AX. Propionic acid is related to the synthesis of cholesterol and influences the glucose metabolism and postprandial blood glucose levels (Cook \& Sellin, 1998).

Until $24 \mathrm{~h}$ of fermentation, none of the fermentation samples showed a significant increase in the concentration of butyric acid. However, at $28 \mathrm{~h}$, the values of C:4 were significantly higher for AXh with a similar value to inulin, but with neglected values for control and AXs. An increase in the production of butyric acid, may result in a protective effect in the distal colon (Wong et al., 2011), since it plays an important role in the regulation of cell proliferation and differentiation (Roberfroid, 2007; Topping \& Clifton, 2001). AXs fermentation might not be considered beneficial in terms of butyric acid production, whereas $\mathrm{AXh}$, which showed molecules with higher molecular weight, displayed a greater increase in butyric acid content.

The SCFA contents were significantly different between WE-AX samples and this might be due to variances in the AXs and AXh structures (Sun et al., 2017). The AXh presented a fraction of bigger molecules (50-450 KDa) which were not found in the soft wheat. In accordance with that, Hughes et al. (2007) performed an in vitro batch fermentation and observed higher concentrations of acetic, propionic, and butyric acid after the addition of high molecular size AX fractions.

\subsection{Bifidobacterium and Lactobacillus abundances}

The fermentation promoted a significantly increase in Lactobacillus abundance only in the inulin sample as expected and Bifidobacterium showed only significant higher concentrations in the AXs sample (Fig. 2). Bifidobacterium promote human health through different pathways (Meulen et al., 2016; Wang, Sun, Cao, \& Wang, 2010). Bifidobacteria presence in human gut can lower the uptake of lipopolysaccharide from the gut lumen, reinforcing tight junctions of epithelial cells. It can also prevent inflammatory diseases, contributing to the equilibrium of the immune system, and intervening in gut-brain axis (Turroni et al., 

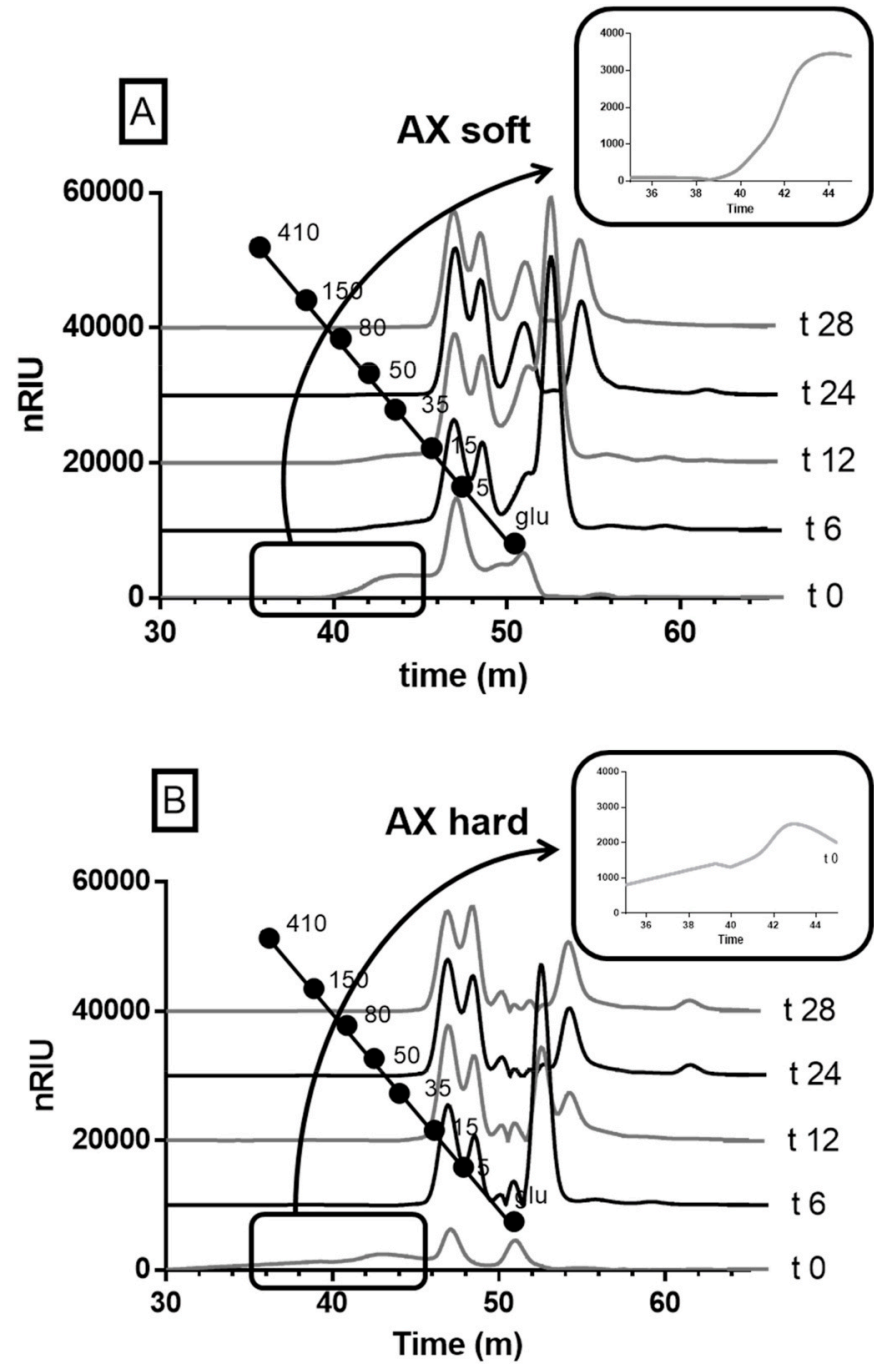

Fig. 3. Variation of the molecular size profile of the WE-AX of the soft (A) and hard (B) wheat during the fermentation with human fecal samples. The curve is made from the average of the measurements made in triplicate. The standard curves are depicted in the graphics and each point represents the peak and the retention time of each standard molecule with a determined molecular weight (kDa) as described in Material and Methods. nRIU: Refractive Index Unit.

2014). In previous study, Aguirre, De Souza, and Venema (2016) illustrated that the fermentation of arabinogalactan and inulin with whole faecal samples from lean and obese subjects increased the abundance of Lactobacillus, and Bifidobacterium. Other researchers have suggested that the increase in the consumption of whole wheat is associated with a higher abundance of Bifidobacterium and Lactobacillus, compared with the intake of refined wheat (Costabile et al., 2008; Rosa-sibakov, 2015).

The increase in Lactobacillus and Bifidobacterium was different in both extracts obtained from soft and hard genotypes. Different wheat pools had possible unequal effects in the microbiome due to bacteria amount and SCFA quantities results, which could be related to the AX structure and composition; this agrees with Gong, Chi, Wang, Zhang, and Sun (2019), who studied the in vitro fermentation of whole and refined wheat flour using human faeces samples.

Bifidobacteria and Lactobacillus can produce xylosidases, $\alpha$-L-arabinofuranosidases (ARF), and xylanases (XYL) for the initial steps of AX fermentation (Crittenden et al., 2002). Because AXs fermentation significantly increased Bifidobacterium abundance, it can be speculated that AXs and AXh have different structures, as has already been described in an earlier work from our group (Paesani et al., 2020). In the case of AXs, it can be metabolised more efficiently than AXh. This ability

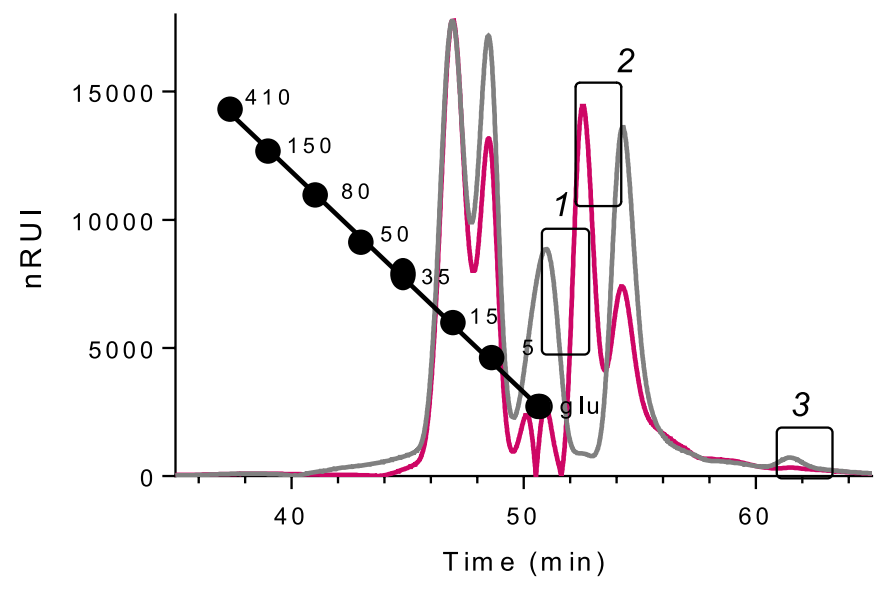

Fig. 4. Differences on the profile of molecular size between WE-AX of the soft arabinoxylans as grey line (AXs) and hard arabinoxylans as pink line (AXh) at $12 \mathrm{~h}$ of the fermentation with human fecal sample. The $12 \mathrm{~h}$ fermentation was the time point with the highest differences in HPSEC profiling for both samples. The squares with numbers indicate the most important differences. The standard curve is depicted in the graphic and each point represents the peak and the retention time of each standard molecule with a determined molecular weight (kDa) as described in Material and Methods. 1) 51 min peak; 2) 53 min peak; 3) 62 min peak. nRIU: Refractive Index Unit. (For interpretation of the references to colour in this figure legend, the reader is referred to the Web version of this article.)

to metabolise $\mathrm{AX}$ is strain dependent and depends on different types of enzymes produced which break the specific AX glycoside bonds. The presence of arabinose substituents could prevent the access of xylanases to hydrolyse the xylose skeleton (Rose et al., 2010). The differences on the WE-AX degradation might be related to xylosidases and ARF produced by Bifidobacteria and xylanases produced by other microorganisms in gut microbiota (Flint, Duncan, \& Scott, 2007; Sun et al., 2017). ARF and XYL enzymes perform very specific hydrolysis of the backbone of $\beta 1-4$ xylose with branched arabinose $1 \alpha-2$ and $1 \alpha-3$ (Correia et al., 2011; McCleary et al., 2015).

\subsection{Variation of the molecular size of the WE-AX during fermentation}

The utilisation of the WE-AX by inoculum was confirmed by the decrease in the molecular weight of these compounds during the fermentation. The first AXs peak found at the beginning of the fermentation ( $t 0$ ) corresponds to the fraction of molecular size between 100 and $20 \mathrm{KDa}$, which decreased significantly after $6 \mathrm{~h}$ and disappeared after $12 \mathrm{~h}$ (Fig. 3). The $49 \mathrm{~min}$ peak appears for the first time at $6 \mathrm{~h}$ of fermentation and corresponds to molecules of approximately $3 \mathrm{KDa}$. The utilisation of AXs can be observed by the higher peak of around $5 \mathrm{kDa}$ ( $47 \mathrm{~min}$ ) after $12 \mathrm{~h}$ of fermentation. The peaks after 52 min (glucose) represent metabolites from fermentation, such as organic acids, with clear differences at various time points.

AXh showed a start of a peak detection at $32 \mathrm{~min}$ and presented two peaks of high molecular weight at $\mathrm{t} 0$, one eluted at 39 and the other at $41 \mathrm{~min}$ (refer to the zoomed pictures inside Fig. 3). Both peaks disappeared completely after $6 \mathrm{~h}$, indicating that the high molecular weight compounds were hydrolysed by the inoculum bacteria. The high molecular weight peaks were not visualised in AXs samples, and a peak detection started at $40 \mathrm{~min}$. In the same way as AXh fermentation, at the time of $6 \mathrm{~h}$, the proportion of the peaks after $40 \mathrm{~min}$ disappeared, and peaks approximately corresponding to $5 \mathrm{KDa}(47 \mathrm{~min})$ and $1 \mathrm{KDa}(51$ min) increased. These peaks were still observed at $12 \mathrm{~h}$ and decreased significantly at 24 and $28 \mathrm{~h}$.

In both cases (AXs and AXh), the overall molecular size of polysaccharides decreased during the fermentation. No changes were observed after $24 \mathrm{~h}$, confirming that fermentation of the AX had already 
been completed. However, after 6 and $12 \mathrm{~h}$, the profiles found for the AX extracts at the same time of fermentation presented different profiles (Fig. 4). On the $12 \mathrm{~h}$ of fermentation, at $51 \mathrm{~min}$, a large peak (1) was observed, and at $62 \mathrm{~min}$, a small one (3) was observed for AXs but not for AXh. In addition, at $53 \mathrm{~min}$, a peak (2) was only observed for AXh. Since theses peaks are below the glucose elution, they are not characterize by carbohydrates but for organic acids formed during fermentation, demonstrating AXs and AXh hydrolysis could have produced different compounds because of different structures. More studies should be done to characterize these compounds.

Hughes et al. (2007) have reported that all fractions of AX showed bifidogenic impact, but this bifidogenic effect was clearly greater as the molecular mass decreased. They have also reported that AX with lower molecular weights were particularly selected for Lactobacillus and $\mathrm{Eu}$ bacterium, and the high molecular weight fractions significantly increased the Bifidobacterium quantities. In our work, the molecular size of WE-AX could have influenced the fermentation speed as well as the selective growth or activity of beneficial intestinal bacteria. AXs fermentation favoured Bifidobacterium growth while AXh increased butyric acid production. The differences found in our study could be due not only to the molecular weight of the extracts but also to the types of linkages of the structure, which can influence bacteria enzyme activity and fermentation. The differences found between the speed and the fermentation capacity of the two AX samples (AXs and AXh), as well as the effects they exert in the intestine, would be due to the different size range of the molecules and the degree of substitution. Since different types of bonds and substitutions are known to exist in arabinoxylans, they can be affected by the action of several different enzymes, including XYL and ARF (Grabber, Hatfield, \& Ralph, 1998; Grabber, Ralph, \& Hatfield, 1998; Sorensen, Pedersen, Jorgensen, \& Meyer, 2007).

\section{Conclusion}

WE-AX from soft wheat showed a prebiotic effect thus possibly while WE-AX from hard wheat produced more butyric acid. This could be due to modulation of the human intestinal microbiota in a beneficial way, but more studies should be done to confirm this. Both WE-AX colonic in vitro fermentation showed a high production of total SCFA, which exert health benefits at local and systemic levels. The use of WE-AX by intestinal bacteria can be verified by the decrease of $\mathrm{pH}$ and the increase of pressure inside flasks, and also the decreasing molecular sizes during colonic fermentation. The different utilisation of the arabinoxylans deriving from various genotypes of wheat reinforces the important influence of the nondigestible carbohydrate source on their chemical structures and the consequent fermentation. The consumption of foods which contain WE-AX (either supplemented or enriched) would have beneficial effects on human health, even on some disorders or diseases characterised by intestinal microbiome dysbiosis. The consumption of WE-AX to maintain the balance of or favourably modulate the profile of the intestinal microbiome can be an important contribution to the quality of human life.

\section{CRediT authorship contribution statement}

Candela Paesani: Methodology, Validation, Formal analysis, Investigation, Writing - original draft, Visualization. Lorena S. Sciarini: Formal analysis, Writing - review \& editing. Malena Moiraghi: Validation, Formal analysis. Emiliano Salvucci: Writing - review \& editing. Samira Prado: Validation, Writing - review \& editing. Gabriela Teresa Pérez: Conceptualization, Writing - review \& editing, Supervision, Project administration, Funding acquisition. João Paulo Fabi: Conceptualization, Writing - review \& editing, Supervision, Project administration, Funding acquisition.

\section{Declaration of competing interest}

The authors declare they have no conflict of interest declared.

\section{Acknowledgements}

This work was supported by CONICET (Consejo Nacional de Investigaciones científicas y Técnicas - \#PUE 22920160100092CO) and the Red Macro Universidades programe. This research was financially supported by grants \#2012/23970-2, \#2013/07914-8 and \#2019/ 11816-8, São Paulo Research Foundation (FAPESP).

\section{Appendix A. Supplementary data}

Supplementary data to this article can be found online at https://doi. org/10.1016/j.lwt.2020.110253.

\section{References}

Aguirre, M., De Souza, C. B., \& Venema, K. (2016). The gut microbiota from lean and obese subjects contribute differently to the fermentation of arabinogalactan and inulin. PloS One, 11(7), 1-18. https://doi.org/10.1371/journal.pone.0159236

Al-sheraji, S. H., Ismail, A., Yazid, M., \& Mustafa, S. (2013). Prebiotics as functional foods : A review. Journal of Functional Foods, 5(4), 1542-1553. https://doi.org/ 10.1016/j.jff.2013.08.009

Bakhtiar, S. M., Leblanc, J. G., Salvucci, E., Ali, A., Martin, R., Langella, P., et al. (2013). Bowel Diseases, 342, 10-17. https://doi.org/10.1111/1574-6968.12111

Buksa, K., Nowotna, A., Praznik, W., Gambuś, H., Ziobro, R., \& Krawontka, J. (2010). The role of pentosans and starch in baking of wholemeal rye bread. Food Research International, 43(8), 2045-2051. https://doi.org/10.1016/j.foodres.2010.06.005

Cook, S. I., \& Sellin, J. H. (1998). Review article: Short chain fatty acids in health and disease. Alimentary Pharmacology \& Therapeutics, 499-507.

Corrêa-Oliveira, R., Fachi, J. L., Vieira, A., Sato, F. T., \& Vinolo, M. A. R. (2016). Regulation of immune cell function by short-chain fatty acids. Clinical and Translational Immunology, 5(4), 1-8. https://doi.org/10.1038/cti.2016.17

Correia, M. A. S., Mazumder, K., Brás, J. L. A., Firbank, S. J., Zhu, Y., Lewis, R. J., et al. (2011). Structure and function of an arabinoxylan-specific xylanase. Journal of Biological Chemistry, 286(25), 22510-22520. https://doi.org/10.1074/jbc. M110.217315

Costabile, A., Klinder, A., Fava, F., Napolitano, A., Fogliano, V., Leonard, C., et al. (2008). Whole-grain wheat breakfast cereal has a prebiotic effect on the human gut microbiota: A double-blind, placebo-controlled, crossover study. British Journal of Nutrition, 99(1), 110-120. https://doi.org/10.1017/S0007114507793923

Crittenden, R., Karppinen, S., Ojanen, S., Tenkanen, M., Ma, J., \& Poutanen, K. (2002). In vitro fermentation of cereal dietary fibre carbohydrates by probiotic and intestinal bacteria. Journal of the Science of Food and Agriculture, 789, 781-789. https://doi. org/10.1002/jsfa.1095

Gibson, G. R. (1999). Nutritional and health benefits of inulin and oligofructose dietary modulation of the human gut microflora using the prebiotics oligofructose and inulin 1. Gut Flora Modulation, 1438-1441.

Gibson, G. R., \& Fuller, R. (2000). Aspects of in vitro and in vivo research approaches directed toward identifying probiotics and prebiotics for human use. Journal of Nutrition, 130(2), 391S-395S. https://doi.org/10.1093/jn/130.2.391s

Gibson, G. R., Hutkins, R., Sanders, M. E., Prescott, S. L., Reimer, R. A., Salminen, S. J., et al. (2017). Expert consensus document: The International Scientific Association for Probiotics and Prebiotics (ISAPP) consensus statement on the definition and scope of prebiotics. Nature Reviews Gastroenterology \& Hepatology, 14(8), 491-502. https://doi.org/10.1038/nrgastro.2017.75

Gong, L., Chi, H., Wang, J., Zhang, H., \& Sun, B. (2019). In vitro fermentabilities of whole wheat as compared with refined wheat in different cultivars. Journal of Functional Foods, 52(November 2018), 505-515. https://doi.org/10.1016/j.jff.2018.11.027

Grabber, J. H., Hatfield, R. D., \& Ralph, J. (1998a). Diferulate cross-links impede the enzymatic degradation of non-lignified maize walls. Journal of the Science of Food and Agriculture, 77(2), 193-200. https://doi.org/10.1002/(SICI)1097-0010(199806)77: $2<193::$ AID-JSFA25>3.0.CO;2-A

Grabber, J. H., Ralph, J., \& Hatfield, R. D. (1998b). Ferulate cross-links limit the enzymatic degradation of synthetically lignified primary walls of maize. Journal of Agricultural and Food Chemistry, 46(7), 2609-2614. https://doi.org/10.1021/ jf9800099

Grootaert, C., Van Den Abbeele, P., Marzorati, M., Broekaert, W. F., Courtin, C. M., Delcour, J. A., et al. (2009). Comparison of prebiotic effects of arabinoxylan oligosaccharides and inulin in a simulator of the human intestinal microbial ecosystem. FEMS Microbiology Ecology, 69(2), 231-242. https://doi.org/10.1111/ j.1574-6941.2009.00712.x

Grubben, M., Van den Braak, C., Essenberg, M., Olthof, M., Tangerman, A., Katan, M., et al. (2001). Effect of resistant starch on potential biomarkers for colonic cancer risk in patients with colonic adenomas A controlled trial. Digestive Diseases and Sciences, 46(4), 750-756.

Hughes, S. A., Shewry, P. R., Li, L., Gibson, G. R., Sanz, M. L., \& Rastall, R. A. (2007). In vitro fermentation by human fecal microflora of wheat arabinoxylans. Journal of 
Agricultural and Food Chemistry, 55(11), 4589-4595. https://doi.org/10.1021/ jf070293g

Jonathan, M. C., Wiechen, P. Van, Souza, C., Schols, H. A., \& Gruppen, H. (2012). In vitro fermentation of 12 dietary fibres by faecal inoculum from pigs and humans. Borne, J. J. G. C. Van Den Food Chemistry, 133(3), 889-897. https://doi.org/10.1016/j. foodchem.2012.01.110.

Karppinen, S., Liukkonen, K., Aura, A. M., Forssell, P., \& Poutanen, K. (2000). In vitro fermentation of polysaccharides of rye, wheat and oat brans and inulin by human faecal bacteria. Journal of the Science of Food and Agriculture, 80(10), 1469-1476. https://doi.org/10.1002/1097-0010(200008)80:10<1469::AID-JSFA675 > 3.0.CO; 2-A

Kasubuchi, M., Hasegawa, S., Hiramatsu, T., Ichimura, A., \& Kimura, I. (2015). Dietary gut microbial metabolites, short-chain fatty acids, and host metabolic regulation. Nutrients, 7(4), 2839-2849. https://doi.org/10.3390/nu7042839

Laar, H. Van, Tamminga, S., Williams, B. A., \& Verstegen, M. W. A. (2002). Fermentation characteristics of polysaccharide fractions extracted from the cell walls of maize endosperm. Journal of the Science of Food and Agriculture, 1375, 1369-1375. https:// doi.org/10.1002/jsfa.1198

McCleary, B. V., McKie, V. A., Draga, A., Rooney, E., Mangan, D., \& Larkin, J. (2015). Hydrolysis of wheat flour arabinoxylan, acid-debranched wheat flour arabinoxylan and arabino-xylo-oligosaccharides by $\beta$-xylanase, $\alpha$-l-arabinofuranosidase and $\beta$-xylosidase. Carbohydrate Research, 407, 79-96. https://doi.org/10.1016/j. carres.2015.01.017

Mendis, M., \& Simsek, S. (2015). Production of structurally diverse wheat arabinoxylan hydrolyzates using combinations of xylanase and arabinofuranosidase. Carbohydrate Polymers, 132, 452-459. https://doi.org/10.1016/j.carbpol.2015.05.083

Meulen, T. A. Van Der, Harmsen, H. J. M., Bootsma, H., Spijkervet, F. K. L., Kroese, F. G. M., \& Vissink, A. (2016). The microbiome - systemic diseases connection. Oral Diseases, 719-734. https://doi.org/10.1111/odi.12472

Paesani, C., Degano, A. L., Salvucci, E., Zalosnik, M. I., Fabi, J. P., Sciarini, L., et al. (2020). Soluble arabinoxylans extracted from soft and hard wheat show a differential prebiotic effect in vitro and in vivo. Journal of Cereal Science, 93, 102956. https://doi.org/10.1016/j.jcs.2020.102956

Paesani, C., Salvucci, E., Moiraghi, M., Fernandez Canigia, L., \& Pérez, G. T. (2019). Arabinoxylan from Argentinian whole wheat flour promote the growth of Lactobacillus reuteri and Bifidobacterium breve. Letters in Applied Microbiology, 68 (2), 142-148. https://doi.org/10.1111/lam.13097

Petrosino, J. F., Highlander, S., Luna, R. A., \& Gibbs, R. A. (2009). Metagenomic pyrosequencing and microbial identification. Clinical Chemistry, 55(5), 856-866. https://doi.org/10.1373/clinchem.2008.107565

Qin, J., Li, R., Raes, J., Arumugam, M., Burgdorf, K. S., Manichanh, C., et al. (2010). A human gut microbial gene catalogue established by metagenomic sequencing. Nature, 464(7285), 59-65. https://doi.org/10.1038/nature08821

Roberfroid, M. (2007). Prebiotics: The concept revisited. Journal of Nutrition, 137(3), 830S-837S. https://doi.org/10.1093/jn/137.3.830s

Roberfroid, M. B., \& Gibson, G. R. (1998). The bifidogenic nature of chicory inulin and its hydrolysis products. Loo, J. A. E. Van. American Society for Nutritional Sciences.

Rosa-sibakov, N. (2015). How does wheat grain, bran and aleurone structure impact their nutritional and technological properties ? Trends in Food Science \& Technology, 41, 118-134. https://doi.org/10.1016/j.tifs.2014.10.003

Rose, D. J., Inglett, G. E., \& Liu, S. X. (2010). Utilisation of corn (Zea mays) bran and corn fiber in the production of food components. Journal of the Science of Food and Agriculture, 90(6), 915-924. https://doi.org/10.1002/jsfa.3915
Ruiz Álvarez, V., Puig Peña, Y., \& Rodríguez Acosta, M. (2010). Microbiota intestinal , sistema inmune y obesidad Intestinal microbiota, immune system and obesity, Revista Cubana de Investigaciones Biomédicas, 29(3), 364-397.

Rumpagaporn, P., Reuhs, B. L., Kaur, A., Patterson, J. A., Keshavarzian, A., \& Hamaker, B. R. (2015). Structural features of soluble cereal arabinoxylan fibers associated with a slow rate of in vitro fermentation by human fecal microbiota. Carbohydrate Polymers, 130, 191-197. https://doi.org/10.1016/j. carbpol.2015.04.041

Salvucci, E. (2014). Microbiome, holobiont and the net of life. Critical Reviews in Microbiology, 7828, 1-10. https://doi.org/10.3109/1040841X.2014.962478

Saulnier, L., Sado, P. E., Branlard, G., Charmet, G., \& Guillon, F. (2007). Wheat arabinoxylans: Exploiting variation in amount and composition to develop enhanced varieties. Journal of Cereal Science, 46(3), 261-281. https://doi.org/10.1016/j. jcs.2007.06.014

Sorensen, H. R., Pedersen, S., Jorgensen, C. T., \& Meyer, A. S. (2007). Enzymatic hydrolysis of wheat arabinoxylan by a recombinant "minimal" enzyme cocktail containing $\beta$-xylosidase and novel endo-1,4- $\beta$-xylanase and $\alpha$-L-arabinofuranosidase activities. Biotechnology Progress, 23(1), 100-107. https://doi.org/10.1021/ bp0601701

Sun, P., Sun, D., \& Wang, X. (2017). Effects of Scutellaria barbata polysaccharide on the proliferation, apoptosis and EMT of human colon cancer HT29 Cells. Carbohydrate Polymers, 167, 90-96. https://doi.org/10.1016/j.carbpol.2017.03.022

Theodorou, M. K., Williams, B. A., Dhanoa, M. S., Mcallan, A. B., \& France, J. (1994). A simple gas production method using a pressure transducer to determine the fermentation kinetics of ruminant feeds. Animal Feed Science and Technology, 48, 185-197.

Topping, D. L., \& Clifton, P. M. (2001). Short-chain fatty acids and human colonic function: Roles of resistant starch and nonstarch polysaccharides. Physiological Reviews, 81(3), 1031-1064. https://doi.org/10.1152/physrev.2001.81.3.1031

Turroni, F., Ventura, M., Buttó, L. F., Duranti, S., O'Toole, P. W., Motherway, M. O. C., et al. (2014). Molecular dialogue between the human gut microbiota and the host: A Lactobacillus and Bifidobacterium perspective. Cellular and Molecular Life Sciences, 71(2), 183-203. https://doi.org/10.1007/s00018-013-1318-0

Van Craeyveld, V., Swennen, K., Dornez, E., Van de Wiele, T., Marzorati, M., Verstraete, W., et al. (2008). Structurally different wheat-derived arabinoxylooligosaccharides have different prebiotic and fermentation properties in rats. Journal of Nutrition, 138(12), 2348-2355. https://doi.org/10.3945/ jn.108.094367

Wang, J., Sun, B., Cao, Y., \& Wang, C. (2010). In vitro fermentation of xylooligosaccharides from wheat bran insoluble dietary fiber by Bifidobacteria. Carbohydrate Polymers, 82(2), 419-423. https://doi.org/10.1016/j. carbpol.2010.04.082

Williams, B. A., Bosch, M. W., Boer, H., Verstegen, M. W. A., \& Tamminga, S. (2005). An in vitro batch culture method to assess potential fermentability of feed ingredients for monogastric diets. Animal Feed Science and Technology, 123-124, 445-462. https://doi.org/10.1016/j.anifeedsci.2005.04.031. Pa.

Williams, M. T., \& Hord, N. G. (2005). The role of dietary factors in cancer Prevention : Beyond fruits and vegetables. Nutrition in Clinical Practice, 451-459. https://doi.org/ 10.1177/0115426505020004451

Wong, J. M., Russel de Souza, R., Cyril, R., Kendall, W., Azadeh Emam, M., \& Jenkis, D. (2011). New prismatic solid-shell element: Assumed strain formulation and hourglass mode analysis. Structural Engineering \& Mechanics, 37(2), 253-256. https://doi.org/10.12989/sem.2011.37.2.253 